\title{
Internet and advertisement
}

\author{
Khaled Moustafa ${ }^{\mathrm{i}}$
}

\begin{abstract}
The Internet has revolutionized the way knowledge is currently produced, stored and disseminated. A few finger clicks on a keyboard can save time and many hours of search in libraries or shopping in stores. Online trademarks with an (e-) prefix such as e-Library, eBusiness, e-Health etc., are increasingly part of our daily professional vocabularies. However, the Internet has also produced multiple negative side effects, ranging from an unhealthy dependency to a dehumanization of human relationships. Fraudulent, unethical and scam practices are also flourishing through for example misleading online advertising methods. Some social and professional networks gather users' profiles for selling and advertising purposes, sometimes by making it technically difficult to unsubscribe. Here, I discuss some of these unethical aspects and propose some potential solutions to reduce them.
\end{abstract}

Keywords: online advertising, misleading advertising, internet misuse, advertising ethics, misleading advertisement, social network, professional network.

The Internet is one of the most impressive inventions in history. The list of online based services is getting longer every day in most professional and entertainment fields. Almost each real life activity has now its virtual counterpart on the Internet by simply adding the prefix (e-) to get an established online equivalent, such as e-commerce, e-book, e-bank, e-library, e-ticket, e-learning, e-shopping, etc.). Indisputably, the Internet has facilitated the communication and the diffusion of knowledge worldwide in an unprecedented way, turning upside down the notion of time and distance. Conversely, the internet has also opened large windows for all kinds of unethical and cheating practices, ranging from dependency and addiction (Shaw and Black 2008) to complex frauds and scams. Entire businesses models have been built online and operate with or without ethical rules. Among these, intemperate and annoying advertising practices where online pages are increasingly flooded with all kinds of advertisements (ads) that dilute the information flow and distract readers from their reading focus. The Internet has become one of the most effective mass platforms for the direct-to-consumer advertising services to advertise any sort of beneficial or harmful, useful or useless products. 
Generally speaking, it might be nothing wrong with the advertising practices, provided truth and honesty are respected. It is also necessary to look on the good side of the advertising as it sometimes pays for the 'free' delivery of useful information, though charges are already covered by other means (VAT, increased sales etc.). Advertising is basically a form of communication and a source of money for advertisers who should usually invest in honest and ethical advertising campaigns, but unfortunately this is not always the case. Due to a lot of intrusive and permanent publicities or inquiries to view flash or video messages, to delay the display of a page's content, to accept cookies (small text files to store information to help advertisers target users' interests)*, or to read dialog boxes or untimely ad windows that open brusquely (hence their common name as 'pop-ups'), the browsing of internet pages is more and more daunting and irritating. The concerns of online advertising appear particularly in the invasive ways that ads are produced and disseminated, often breaking some fundamental ethical rules. It is very common, for example, that Internet's users are frequently and suddenly solicited against their will to fill in online consumption surveys, to view pop-ups, to hear audio contents or to accept cookies. Some websites even force visitors to stay on an Internet page to view ads' videos of about 30 seconds or so, before offering the possibility to skip the ad page and move forward to the requested content.

Some social and professional networks follow similar forcing practices and make the unrolling process technically intimidating so members are discouraged to unsubscribe and remain potential targetable consumers. The rationale behind this practice is that, the more members registered, the more potential consumers targeted and then the more money will be collected. With such unethical practices, however, social networks would infringe users' autonomy and privacy (O'Brolchain, Jacquemard et al. 2015). Examples of misleading advertising practices exist in almost all domains. In the medical and health care sector, for example, misleading advertising practices are particularly problematic with negative repercussions on the society. The debate about the prohibition for advertising in cosmetic surgery is still controversial (Fatah 2012) (Taber 2012), though surgeons' leaders opt for banning such advertising (O'Dowd 2012). In the high-tech products industry, the technical specifications of advertised products are sometimes falsely enticing. Technical computer characteristics, for example, might display that the hard disk storage space is, say, 1000 GB (Giga Bytes), but practically the real space available would be less, actually closer to $~ 930 \mathrm{~GB}$ or so. A difference of about $\sim 70 \mathrm{~GB}$ is thus missing between what is advertised and what is really available. Such differences are often justified by the fact that the operating system files occupy some space, which is true, but in such cases one may wonder why, then, not advertise the truly available space after the deduction of the hard disk space occupied by the system's files? Computer manufacturers should either add an extra 100 GB (or whatever other storage sizes allocated 
specifically for the system's files), or alternatively advertise only the remaining storage value that provides accurate information. Another trivial advertising example comes from smartphone manufactures/users when emails sent from smartphones are often appended with a default statement saying that the email was 'Sent from my smartphone's brand' to advertise the smartphone brand!

More misleading advertising practices are made with food or drink products. For example, a food advertiser on TV or Internet often shows an 'immediate appreciation' of an advertised food product as soon as $s /$ he gets a mouthful while $s /$ he has merely tasted it to judge its deliciousness or appetizing quality! In other word, the immediate appreciation of the advertised food is untrue and not based on real or natural circumstances. Although parents are generally immune to such misleading tricks, children would fall easily victim to such false advertising subtleties because they are not always able to recognize the deceptive nature of advertising practices (Lioutas and Tzimitra-Kalogianni 2014) (Pettigrew, Tarabashkina et al. 2013), which are generally intended to promote products rightly or wrongly.

In many cases, advertising is fanciful with surrealistic illustrations or irrational imaginative contents. As consumers, we can wonder if not all advertising is somewhat misleading as it only focuses on emphasising the supposedly good and beneficial aspects of the goods in question while leaving out any potential defects and deficiencies. In fact, advertisers rarely highlight the potential defects of their advertised products because if they do so, they would not attain their commercial objective in promoting their goods and growing their business.

Further pitfalls associated with advertising and intensive internet uses, in particular virtual networking, deeply influence the social and personal behaviors. A high level of online activity for example on Twitter (Clayton 2014) or Facebook (Clayton, Nagurney et al. 2013) would be associated with negative relationship outcomes on the emotional and familial conflicts. Another study reports that Facebook addiction may under certain conditions be linked to a form of dissatisfaction in human relationships due to the jealousy behavior it produces (Elphinston and Noller 2011).

To reduce unethical publicity issues, particularly for health care services, advertising needs to be regulated (Masoni, Guelfi et al. 2011). This regulation should be done in a way that the advertising messages should conform to the real effects and values of the advertised products. Advertisers should also be aware that ethically questionable ads can produce negative effects, opposite to what they would expect, which may damage the reputation of their brand or products. Advertisers should comply with ethical advertising guidelines to insure that their 
advertisements are truly and honestly produced to serve the public and gain its loyalty. The direct-to-consumer advertising approaches should also be built on transparency and robustness (Mackey and Liang 2015), and Internet users should not be constrained to view ads or to be penalized by long waits in watching unwanted ads before obtaining the requested page's content. Instead, users should have full control over the kind of ads they wish to filter or to receive.

Among the other potential solutions to standardize online advertising might be the development of special Internet browsers intended explicitly for advertising purposes only, and other browsers designed for professional uses only without any intrusive or invasive advertising. Some media or news channels already ban advertising practices in their programs and so they are often delineated as serious and trustful sources of information. Similarly, specialized websites or internet browsers dedicated specifically to advertising purposes might be developed to keep serious websites and educational sources clean and devoid from any advertisement activity. People interested in commercial advertising can then look for those advertisements that are specific to their interests.

Disclosure: None.

\section{Reference}

Clayton, R. B. (2014). "The third wheel: the impact of Twitter use on relationship infidelity and divorce." Cyberpsychol Behav Soc Netw 17(7): 425-430.

Clayton, R. B., A. Nagurney and J. R. Smith (2013). "Cheating, breakup, and divorce: is Facebook use to blame?" Cyberpsychol Behav Soc Netw 16(10): 717-720.

Elphinston, R. A. and P. Noller (2011). "Time to face it! Facebook intrusion and the implications for romantic jealousy and relationship satisfaction." Cyberpsychol Behav Soc Netw 14(11): 631-635.

Fatah, F. (2012). "Should all advertising of cosmetic surgery be banned? Yes." BMJ 345: e7489.

Lioutas, E. D. and I. Tzimitra-Kalogianni (2014). "'I saw Santa drinking soda!' Advertising and children's food preferences." Child Care Health Dev.

Mackey, T. K. and B. A. Liang (2015). "It's Time to Shine the Light on Direct-to-Consumer Advertising." Ann Fam Med 13(1): 82-85.

Masoni, M., M. R. Guelfi and G. F. Gensini (2011). "Online healthcare advertising needs regulation." BMJ 342: c5778. 
O'Brolchain, F., T. Jacquemard, D. Monaghan, N. O'Connor, P. Novitzky and B. Gordijn (2015). "The Convergence of Virtual Reality and Social Networks: Threats to Privacy and Autonomy." Sci Eng Ethics.

O'Dowd, A. (2012). "Surgeons' leaders call for ban on cosmetic surgery advertising." BMJ 344: e627.

Pettigrew, S., L. Tarabashkina, M. Roberts, P. Quester, K. Chapman and C. Miller (2013). "The effects of television and Internet food advertising on parents and children." Public Health Nutr 16(12): 2205-2212.

Shaw, M. and D. W. Black (2008). "Internet addiction: definition, assessment, epidemiology and clinical management." CNS Drugs 22(5): 353-365.

Taber, S. (2012). "Should all advertising of cosmetic surgery be banned? No." BMJ 345: e7508.

* http://windows.microsoft.com/en-us/windows/cookies-faq\#1TC=windows-7 Accessed on 20 Mars $\underline{2015}$

'Email: khaled.moustafa@gmail.com 